\title{
SOLUTION OF PLANAR DIOPHANTINE EQUATIONS
}

\author{
JAMES C. OWINGS, JR.
}

\begin{abstract}
We continue our investigation, begun in [2], of the "planes" associated with the Diophantine equation

$$
x^{2}+y^{2}+z^{2} \pm y z \pm x z \pm x y+g x+h y+k z+m=0 .
$$

In particular, we show that the number of planes is finite, thus providing a new way of finding all integral solutions of this equation. Hopefully, our methods will extend to a treatment of the general quadratic equation in three variables.
\end{abstract}

In [2] we introduced the notion of "planar" for quadratic Diophantine equations in three variables and solved the special case $x^{2}+y^{2}+z^{2}-y z-$ $x z-x y-x-2 y-3 z=0$. In this paper we will extend the methods of [2] to solve all planar equations, proving along the way certain theorems useful in solving certain nonplanar equations as well.

We first recapitulate the notion of "planar". Consider the general 3variable quadratic equation:

$$
a x^{2}+b y^{2}+c z^{2}+d y z+e x z+f x y+g x+h y+k z+m=0 .
$$

If $(x, y, z)$ is a solution, one easily verifies that $\left(x^{\prime}, y, z\right),\left(x, y^{\prime}, z\right)$, and $\left(x, y, z^{\prime}\right)$ are also solutions, where $x^{\prime}, y^{\prime}$, and $z^{\prime}$ are defined by:

$$
\begin{aligned}
& a x^{\prime}=-a x-f y-e z-g, \\
& b y^{\prime}=-b y-f x-d z-h, \\
& c z^{\prime}=-c z-e x-d y-k .
\end{aligned}
$$

So if one starts with a solution

$$
\begin{array}{lll} 
& x & \\
& & z
\end{array}
$$

one can expand to the configuration

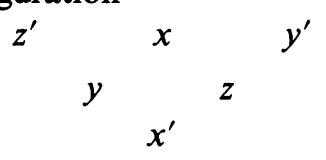

FIGURE 1

in which every small equilateral triangle of numbers represents a solution to the equation, once the proper association is made between numbers and variables. This process may be continued so as to generate an infinite "family" of solutions. Hence, solving equation (1) is reduced to the

Received by the editors October 12, 1977 and, in revised form, December 6, 1977.

AMS (MOS) subject classifications (1970). Primary 10B05.

- American Mathematical Society 1978 
determination of how many such families there are and producing a member of each. This approach to quadratic Diophantine equations was initiated in [1] by W. H. Mills, who, however, was only considering two variables. In his case, the "families" could easily be visualized as endless chains

$$
\ldots y^{\prime} \quad x \quad y \quad x^{\prime} \ldots
$$

It is tempting for us to visualize our families as triangular lattices covering the plane. However, there may be a problem.

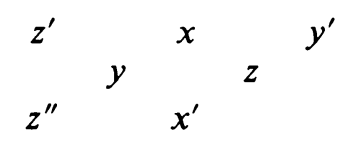

FIGURE 2

Suppose, starting with Figure 1, one calculates $z^{\prime \prime}$, as in Figure 2, and wishes to know what number should occupy the position marked in Figure 2 by the dot. It can be computed in two different ways, either from the solution $\left(x, y, z^{\prime}\right)$ or from the solution $\left(x^{\prime}, y, z^{\prime \prime}\right)$. "Planarity" demands that these two methods yield the same answer. As shown in [2], the corresponding algebraic condition is $e^{2}=a c$; similar considerations involving the other two variables yield $f^{2}=a b$ and $d^{2}=b c$. When one imposes the additional restriction that $x^{\prime}, y^{\prime}$, and $z^{\prime}$ be integers whenever $x, y$, and $z$ are integers, one finds that, certain trivial cases excepted, all planar equations have the form

$$
x^{2}+y^{2}+z^{2} \pm x y \pm x z \pm y z+g x+h y+k z+m=0
$$

where $g, h, k, m$ are integers. In other words, $a=b=c=1$ and $|d|=|e|=$ $|f|=1$. It is this equation that we solve, giving an efficient algorithm for deciding when it has solutions and for generating all "planes" of solutions when they exist. It will turn out that the number of planes is always finite.

We first isolate those cases of (2) which have only finitely many solutions. If one starts with (1) and completes the square on $x$ and then on $y$, one gets the following equation, equivalent to the original whenever $4 a b-f^{2} \neq 0$.

$$
\begin{aligned}
\left(4 a b-f^{2}\right)(2 a x+ & f y+e z+g)^{2} \\
& +\left[\left(4 a b-f^{2}\right) y+(2 a d-f e) z+2 a h-f g\right]^{2} \\
& +4 a\left(c\left(4 a b-f^{2}\right)+d(f e-a d)-b e^{2}\right) z^{2} \\
& +4 a\left(4 a b k+d f g-2 a d h-2 b e g-k f^{2}+f e h\right) z \\
& +4 a\left(4 a b m-b g^{2}-a h^{2}-m f^{2}+f g h\right)=0 .
\end{aligned}
$$

This leads immediately to the following fact.

TheOREM 1. If $4 a b-f^{2}>0$ and $a c\left(4 a b-f^{2}\right)+a d(f e-a d)-a b e^{2}>0$, then equation (1) has only finitely many solutions $(x, y, z)$.

For planar equations the first condition becomes $4 \cdot 1 \cdot 1-( \pm 1)^{2}>0$, or $3>0$. The second condition becomes $3+\operatorname{def}-1-1>0$, or def $>-1$. 
Therefore, since $d= \pm 1, e= \pm 1$, and $f= \pm 1$, a planar equation will have finitely many solutions whenever def $=+1$. Suppose we had $d=1, e=1$, $f=-1$. Perform the substitution $u=-z$. Our equation then becomes $x^{2}+y^{2}+u^{2}-y u-x u-x y+g x+h y-k u+m=0$. Therefore, to solve all planar equations we need only consider the case $d=e=f=-1$, that is, the following equation:

$$
x^{2}+y^{2}+z^{2}-y z-x z-x y+g x+h y+k z+m=0 .
$$

Before analyzing this equation, we first make a definition and prove the central theorem of our paper, a general fact about equation (1).

Definition. A source is a solution $(x, y, z)$ to equation (1) such that $x^{\prime} \geqslant x$, $y^{\prime} \geqslant y$, and $z^{\prime} \geqslant z$, where $x^{\prime}, y^{\prime}$, and $z^{\prime}$ are defined by equations (A).

THeOREM 2. Suppose there exists a constant $M$ and a function $F$ such that every solution $(x, y, z)$ to equation (1) satisfies $x \geqslant M, y \geqslant F(x)$. Then any family of solutions must contain at least one source.

Proof. (cf. p. 268 of [2]). Assume a family exists which does not contain a source and let $x$ be the smallest $x$-value occurring in it. Choose $y$ and $z$ such that $(x, y, z)$ is a solution in the family and $y$ is as small as possible (it cannot be less than $F(x))$. From the solution $(x, y, z)$ compute $x^{\prime}, y^{\prime}$, and $z^{\prime}$ using equations (A). Then $\left(x^{\prime}, y, z\right),\left(x, y^{\prime}, z\right)$, and $\left(x, y, z^{\prime}\right)$ are also in the family. Again, using equations (A), compute $x^{\prime \prime}$ and $y^{\prime \prime}$ from $\left(x, y, z^{\prime}\right)$ such that $\left(x^{\prime \prime}, y, z^{\prime}\right)$ and $\left(x, y^{\prime \prime}, z^{\prime}\right)$ are in the family (see Figure 3$)$.

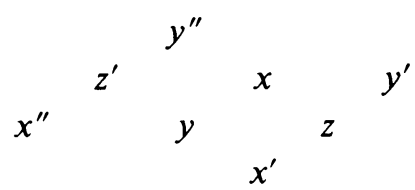

FIGURE 3

Since $x$ and $y$ were minimized, $x \leqslant x^{\prime}$ and $y \leqslant y^{\prime}$. Therefore, because $(x, y, z)$ is not a source, $z>z^{\prime}$. So, since $\left(x, y, z^{\prime}\right)$ is not a source and $x \leqslant x^{\prime \prime}$, we conclude that $y>y^{\prime \prime}$, a contradiction.

We now return to (4). Notice that we can assume $g+h+k \leqslant 0$, for if this is not true we make the substitution $u=-x, v=-y, w=-z$. Since $d=e=f=-1$, we find by (3) that (4) is equivalent to:

$$
\begin{aligned}
& 3(2 x-y-z+g)^{2}+(3(y-z)+2 h+g)^{2} \\
& \quad=-12(g+h+k) z+4\left(g^{2}+h^{2}+g h\right)-12 m .
\end{aligned}
$$

If $g+h+k=0$, the substitution $u=2 x-y-z+g, v=3(y-z)+$ $2 h+g$ transforms (5) into $3 u^{2}+v^{2}=4\left(g^{2}+h^{2}+g h\right)-12 m$, which has finitely many solutions in $u, v$. It is easy to verify that, whenever a solution plane contains $(x, y, z)$ it also contains $(x+u, y+u, z+u)$ and $(x-u, y$ $-u, z-u)$ where, as above, $u=2 x-y-z+g$. Since $\left(x_{1}, y_{1}, z_{1}\right)$ and $\left(x_{2}, y_{2}, z_{2}\right)$ give the same values to the variables $u$ and $v$ iff, for some integer 
$q,\left(x_{2}, y_{2}, z_{2}\right)=\left(x_{1}+q, y_{1}+q, z_{1}+q\right)$, it follows that there are finitely many planes.

If $g+h+k<0$, it is immediately clear from (5) that $z$ is bounded from below. By symmetry there exists a constant $M$ such that, for any solution $(x, y, z), x \geqslant M, y \geqslant M$, and $z \geqslant M$. Theorem 2 tells us that any plane of solutions to (4) must contain a source. We now show that, supposing $g+h+k<0$, (4) can have but finitely many source solutions.

From equations (A) we get the following formulas for $x^{\prime}, y^{\prime}$, and $z^{\prime}$ :

$$
\begin{aligned}
& x^{\prime}=-x+y+z-g, \\
& y^{\prime}=-y+x+z-h, \\
& z^{\prime}=-z+x+y-k .
\end{aligned}
$$

Therefore, $x^{\prime} \geqslant x, y^{\prime} \geqslant y, z^{\prime} \geqslant z$ become:

$$
\begin{aligned}
& x+y+z+(-3 x-g) \geqslant 0, \\
& x+y+z+(-3 y-h) \geqslant 0, \\
& x+y+z+(-3 z-k) \geqslant 0 .
\end{aligned}
$$

Let $s=-(g+h+k)$. Then the left-hand sides of (C) sum to $s$, a positive integer. So the left-hand sides represent three nonnegative integers between 0 and $s$, which means that the difference between any two of them has absolute value at most $s$. Subtracting the second from the first, the third from the second, and the first from the third, we get:

$$
\begin{aligned}
& -s \leqslant(h-g)+3(y-x) \leqslant s, \\
& -s \leqslant(k-h)+3(z-y) \leqslant s, \\
& -s \leqslant(g-k)+3(x-z) \leqslant s .
\end{aligned}
$$

Upon rewriting these become:

$$
\begin{aligned}
& \frac{k+2 g}{3} \leqslant y-x \leqslant \frac{-k-2 h}{3}, \\
& \frac{g+2 h}{3} \leqslant z-y \leqslant \frac{-g-2 k}{3}, \\
& \frac{h+2 k}{3} \leqslant x-z \leqslant \frac{-h-2 g}{3} .
\end{aligned}
$$

Looking back at (5) and observing that $2 x-y-z=(x-y)+(x-z)$, we see that equations (D) imply that the left-hand side of (5) is bounded for solutions that are sources. So, since $g+h+k<0$, we know from the right-hand side of (5) that $z$ is bounded in absolute value, and we can easily compute the bound. Equations (D) now tell us that there are only finitely many sources. So, because each plane must contain a source and is determined by any source it contains, there are only finitely many planes.

For an illustrative example, we refer the reader to $\$ 3$ of [2], where the special case $x^{2}+y^{2}+z^{2}-y z-x z-x y-x-2 y-3 z=0$ was worked 
out before we had developed the general method presented here. That particular equation has two sources, $(0,0,0)$ and $(-1,1,1)$, which belong to different planes. We believe this to be the general situation, i.e., each plane has just one source, but so far we do not have a proof.

\section{REFERENCES}

1. W. H. Mills, A method for solving certain Diophantine equations, Proc. Amer. Math. Soc. 5 (1954), 473-475.

2. J. C. Owings, Jr., An elementary approach to Diophantine equations of the second degree, Duke Math. J. 37 (1970), 261-273.

Department of Mathematics, University of Maryland, College Park, Maryland 20742 